\title{
Compétitivité territoriale : perspectives sur les asymétries régionales au Portugal
}

\author{
Tiago $\mathrm{N}$ eves Sequeira ${ }^{1}$ \\ Université de Beira Interior \\ Covilhã, Portugal
}

\section{Introduction}

La spécialisation et la complémentarité sont de plus en plus considérées comme des concepts clés de la compétitivité territoriale, mesurée par la capacité de susciter des investissements privés et d'attirer une main-d'oeuvre qualifiée. Dans un pays comme le Portugal, les villes de l'intérieur font face à deux nouveaux enjeux avec la mondialisation : profiter des synergies découlant des complémentarités territoriales et développer une nouvelle perspective organisationnelle et informationnelle de l'espace urbain. En effet, une urbanisation vigoureuse est à l'oeuvre dans les régions périphériques, ce qui nous oblige à concevoir plus adéquatement l'articulation des territoires ruraux et des territoires urbains dans ces régions.

\section{Hiérarchie, spécialisation et compétitivité}

Camagni $^{2}$ a identifié cinq principes d'organisation des villes : l'accessibilité, l'agglomération, l'interaction spatiale, la hiérarchie et la compétitivité. Dans la présente étude, nous traiterons de deux de ces principes, soit la hiérarchie et la compétitivité. Selon le même auteur, la ville doit être compétitive en fonction de quatre facteurs : 1) la hiérarchie à laquelle elle appartient; 2) les biens qui constituent sa vocation traditionnelle spécifique; 3) l'intégration des processus productifs intra-industriels; 4) l'intégration horizontale qui engendre des synergies.

De nombreux critères servent à construire des hiérarchies de villes, notamment le critère démo- graphique. Plusieurs auteurs $\mathrm{s}^{3}$ toutefois défendent que la hiérarchie peut le mieux se définir à partir des critères suivants : les fonctions urbaines exercées, les équipements disponibles, les activités économiques et le type de relations et de flux établis avec d'autres villes ou territoires. En somme, nous disons que les villes entrent en compétition en fonction de leurs positions relatives.

Selon Domingues ${ }^{4}$, la compétitivité est avant tout un concept relationnel. L'application de ce concept aux villes se justifie pleinement par le changement profond qui s'est accompli dans la majorité des villes. Elles perdent peu à peu leur importance en tant que centres qui organisent des territoires proches et qui détiennent un haut degré d'autonomie. Les villes ont laissé leur rôle de points fixes d'attraction et de répulsion d'activités et de populations pour devenir des noeuds de relations dans un «espace de flux» marqué par une mobilité de plus en plus grande.

Dans ce contexte théorique, les avantages compétitifs dériveront des spécificités de chaque ville, en tant que lieu d'agglomération des ressources stra tégiques et d'externalités favorables à une consolidation de vrais milieux innovateurs munis d'une capacité de réponse à des changements de plus en plus imprévisibles. En résumé, au-delà des attributs de chaque ville, les liens avec l'environnement et l'atmosphère vécue dans l'espace urbain sont devenus, malgré la difficulté de les mesurer, des facteurs inducteurs de compétitivité. 
Selon Domingues, la compétitivité est avant tout un concept relationnel. L'application de ce concept aux villes se justifie pleinement par le changement profond qui s'est accompli dans la majorité des villes. Elles perdent peu à peu leur importance en tant que centres qui organisent des territoires proches et qui détiennent un haut degré d'autonomie. Les villes ont laissé leur rôle de points fixes d'attraction et de répulsion d'activités et de populations pour devenir des noeuds de relations dans un «espace de flux» marqué par une mobilité de plus en plus grande.

\section{Dimension et compétitivité}

Le concept de ville moyenne ou intermédiaire suggère que les villes ainsi classifiées parcourent une trajectoire. Ce mouvement leur donne une perspective optimiste d'affirmation et les oriente vers de nouveaux contextes relationnels qui peuvent créer des conditions pour de nouvelles «centralités».

La dimension des villes a depuis longtemps été conçue comme liée à une échelle d'optimisation des coûts, le parallèle pouvant être fait avec la détermination de la fonction de production d'une entreprise. Mais dans l'actuel paradigme de production, il y a un changement de l'importance relative des coûts de production. Aussi bien dans les entreprises que sur le plan urbain, on peut dire que l'importance relative de la taille démographique diminue par rapport à l'importance des flux relationnels. L'organisation des complémentarités territoriales et des mobilités avec les territoires voisins devient plus poussée et assure un plus haut niveau de vie aux habitants que la simple dimensions.

Au-delà des attributs de chaque ville, les liens avec l'environnement et l'atmosphère vécue dans l'espace urbain sont devenus, malgré la difficulté de les mesurer, des facteurs inducteurs de compétitivité.

\section{Avantages comparatifs et compétitifs}

Si l'on admet que la dimension n'est pas le principal déterminant de la compétitivité territoriale, on est obligé de le chercher ailleurs, par exemple dans le nouveau paradigme de recomposition résultant de la mondialisation.

Depuis l'oeuvre de Heckscher et de Ohlin ${ }^{6}$, plusieurs auteurs lient les avantages comparatifs avec la dotation relative de facteurs disponibles en chaque pays. En ce sens, on peut appliquer les mécanismes de la spécialisation internationale au niveau régional. Selon Porter? :

«Ce qu'on doit chercher, ce sont les caractéristiques décisives pour une nation qui permettent à ses entreprises de créer et maintenir l'avantage compétitif dans certains champs, soit les avantages compétitifs des nations. [...] L'avantage compétitif est créé et maintenu à travers un processus fortement localisé. [...] Les différences dans les structures économiques, les valeurs, la culture, les institutions et l'histoire nationale contribuent fortement au succès compétitif. [...] Ces idées et concepts théoriques peuvent facilement être adaptés à des unités politiques ou géographiques de moindre dimension que la nation entière. [...] Les politiques de niveau régional ou municipal jouent un rôle important dans la formation de l'avantage national.»

C'est dans ce contexte théorique que la présente étude a été menée. Elle cherche à identifier les spécificités du territoire, élargissant ainsi le concept de facteur productif. Comme exemple concret, on peut dire qu'une ville dont la population est relativement cultivée et qualifiée, où existent des universités et des centres technologiques, doit savoir profiter de cet avantage pour attirer des entreprises innovatrices. En résumé, nous proposons que, pour une ville qui veut améliorer sa production à partir d'une ressource dont elle dispose en abondance, c'est l'intensité d'utilisation de cette dernière qui importe le plus. 


\section{B rèves considérations méthodologiques}

L'absence de statistiques officielles à l'échelle des concelhos (mot portugais désignant le territoire d'une mairie) correspondant aux variables consommation, revenu, exportation, qualification et innovation rend difficile l'identification des spécialisations et dynamiques compétitives. Pour cette raison, nous avons eu recours à l'analyse factorielle pour repérer, au moyen de corrélations spatiales, des facteurs compétitifs.

Nous avons utilisé la méthode de rotation non orthogonale oblimin pour obtenir les hiérarchies, et la méthode de rotation orthogonale varimax pour estimer les spécialisations de chaque concelho par facteur de compétitivité. Une fois déterminés la hiérarchie et les indicateurs de spécialisation par facteur, nous avons eu recours à une analyse de variance (ANOVA) pour identifier les facteurs de compétitivité qui se présentaient de façon significativement différente sur le littoral et à l'intérieur de l'une des régions du pays.

\section{Étude du cas de la région Centro du Portugal}

La région Centro est l'une des cinq régions de planification utilisées par l'État portugais à des fins d'aménagement du territoire. La région Centro a été, pour les fins de l'étude, partagée en deux sous-régions : à l'est, la Beira Litoral, qui touche l'Atlantique, et à l'ouest, la Beira Interior, qui jouxte l'Espagne. Un massif montagneux, la Serra da Estrela, forme une barrière naturelle entre les deux sous-régions. Furent sélectionnés les concelhos avec une population résidante de plus de 2300 habitants comprenant au moins une localité de plus de 2000 habitants.

Selon l'extension de la méthodologie de Porter aux territoires, nous avons groupé les facteurs de compétitivité dégagés par l'analyse factorielle en trois catégories :

\section{1) Facteurs basiques de compétitivité}

Pouvoir d'achat, emploi, services et tourisme;

\section{2) Facteurs avancés de compétitivité}

Qualification de la main-d'oeuvre et services supérieurs, dynamisme des entreprises, effet d'échelle et internationalisation;
3) Compétitivité relative à l'environnement urbain Urbanité/densité, sécurité urbaine et sécurité routière.

Les hypothèses qui ont été formulées sont les suivantes :

1) Les principales villes de la Beira Litoral assument une prépondérance dans les hiérarchies établies;

2) Il existe des spécialisations différentes par facteur de compétitivité dans les villes étudiées;

3) On peut établir des avantages ou des désavantages absolus et comparatifs de l'ensemble des villes de Beira Litoral vis-à-vis l'ensemble des villes de Beira Interior et vice versa.

\section{Résultats}

De l'application de cette méthodologie, il ressort une évidente suprématie des performances des villes de la Beira Litoral, ce qui est tout à fait cohérent avec la perception de tous les observateurs de la réalité portugaise.

\section{Hiérarchie}

De la première analyse, nous avons obtenu quatre facteurs : secteur tertiaire-consommation, emploi, tourisme et pouvoir d'achat. De la seconde analyse, nous avons pu extraire les deux facteurs suivants : qualification de la main-d'oeuvre et services supérieurs; dynamisme des entreprises, effet d'échelle et internationalisation de l'économie locale. De la troisième analyse, trois facteurs on été dégagés : urbanité/densité, sécurité urbaine et sécurité routière.

En résumé, nous pourrions dire que dans la première analyse :

1) le facteur «tertiaire-consommation» nous indique la dotation en services du concelho. Ce facteur est également associé à des variables de consommation;

2) le facteur «emploi» traduit la capacité d'offre d'emplois aux habitants du concelho; 


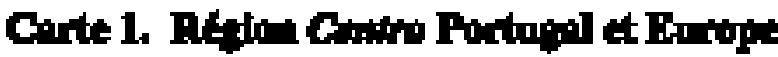
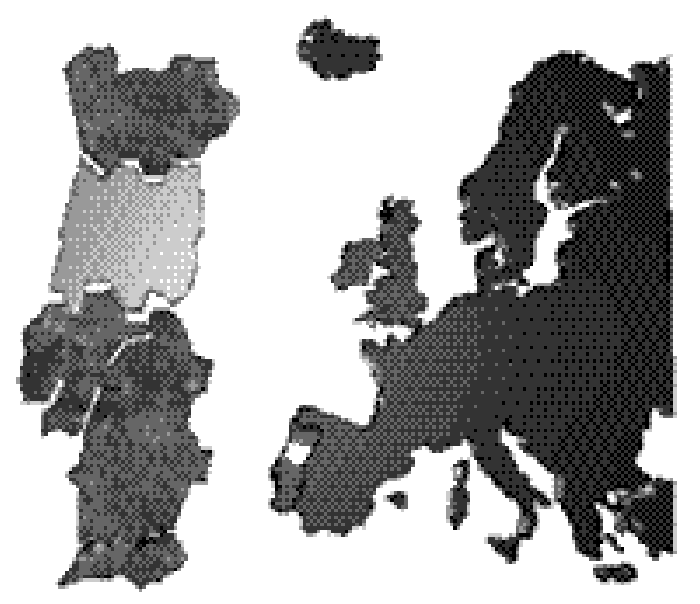

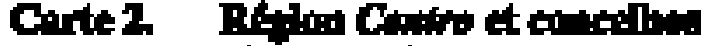 etectilinat.}

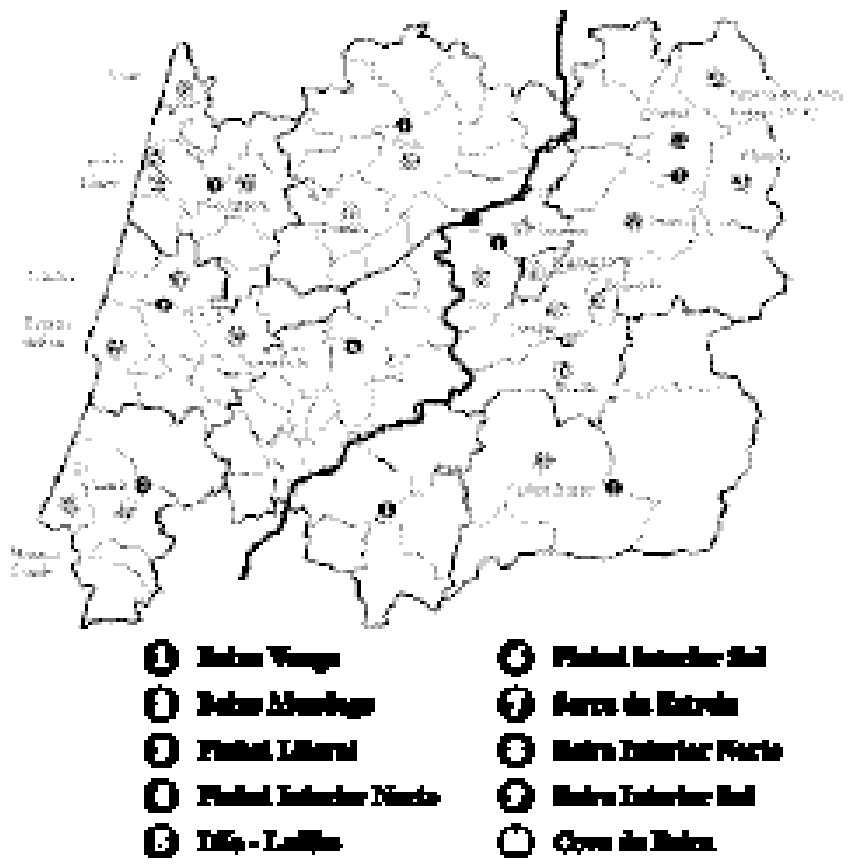

3) le facteur «tourisme» reflète l'importance du tourisme dans une optique mixte entre demande et offre;

4) le facteur «pouvoir d'achat» peut être considéré comme une variable proxy du pouvoir d'achat et peut servir en tant que mesure de capacité à attirer des cadres qualifiés.
En ce qui concerne la seconde analyse, nous pourrions dire que :

1) le facteur «qualification de la main-d'œuvre et services supérieurs» exprime l'importance relative d'indicateurs tels que l'enseignement, la recherche, la santé et la culture;

2) le facteur «dynamisme des entreprises, effet d'échelle et internationalisation de l'économie locale» traduit le dynamisme des entreprises, leur échelle de fonctionnement et leur capacité d'internationalisation.

Au cours de la troisième analyse, nous avons dég agé les facteurs suivants :

1) le facteur «urbanité/densité», qui sert d'indicateur des caractéristiques plus ou moins urbaines des concelhos;

2) le facteur «sécurité urbaine», qui est en relation avec le nombre d'agressions et avec l'insécurité urbaine;

3) le facteur «sécurité routière », qui est lié de près au nombre d'accidents et à la congestion du trafic sur les routes.

Il faut ajouter que toutes les variables sont exprimées en termes relatifs, ceci pour respecter le principe selon lequel la compétitivité doit être mesurée en relation avec le territoire en soustrayant l'effet dimension. Cette option méthodologique soulève des problèmes concrets, surtout quand il s'agit d'analyser des marchés où la demande est supérieure à ce que permet le niveau de la population, notamment l'enseignement supérieur, le tourisme et l'industrie. La compétitivité d'un territoire dans une certaine activité n'est stratégiquement pertinente que si, dans les faits, elle peut représenter un poids significatif dans son économie. Malgré tout, considérant que le but de l'étude était l'analyse de la spécialisation, nous avons accepté de courir le «risque» de favoriser les concelhos de petite dimension pour certains facteurs.

\section{Spécialisation}

Dans cette partie de l'étude, nous voulons déterminer les facteurs de compétitivité sur lesquels chaque 
concelho devrait miser davantage en vue de minimiser son coût d'opportunité. Et l'interprétation la plus simple sera de conseiller la spécialisation dans les activités liées aux facteurs relativement plus abondants, parce que de cette façon, le coût d'opportunité se trouve minimisé. La représentation cartographique qui suit traduit les résultats de l'étude de façon éloquente.

Nous voulons déterminer les facteurs de compétitivité sur lesquels chaque concelho devrait miser davantage en vue de minimiser son coût d'opportunité. Et l'interprétation la plus simple sera de conseiller la spécialisation dans les activités liées aux facteurs relativement plus abondants, parce que de cette façon, le coût d'opportunité se trouve minimisé.

\section{Standard territorial de spécialisation}

\section{Carte 3. Facteurs basiques}

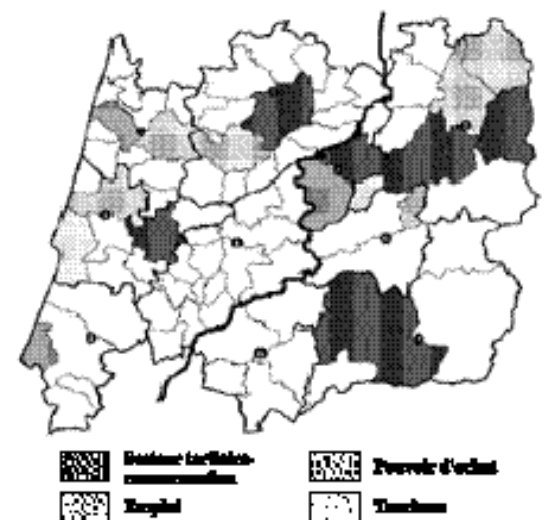

Carte 4. Facteurs avancés

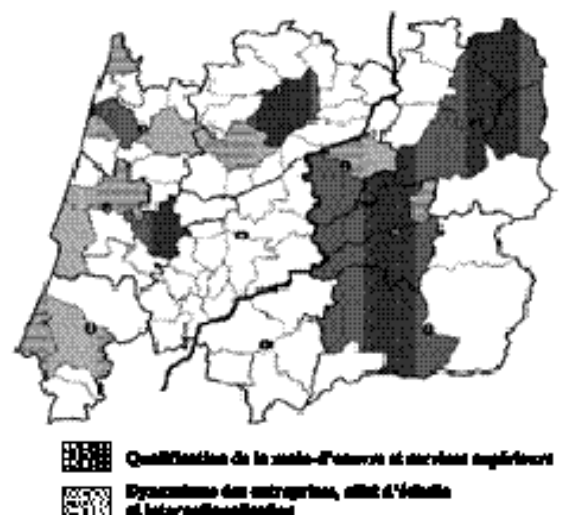

Carte 5. Facteurs d'environnement

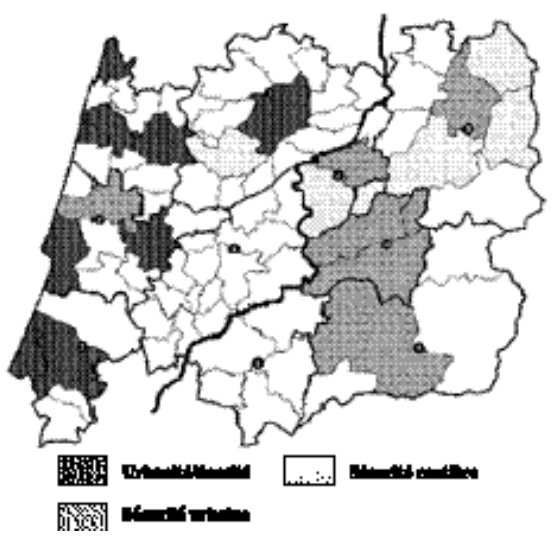

Avantages compétitifs

En suivant la taxonomie définie, la Beira Litoral présente des avantages absolus pour quatre facteurs sur dix et la Beira Interior pour les deux facteurs de sécurité, sans doute associés à une plus faible densité démographique. Le tableau 1 qui suit présente une synthèse des principales conclusions de l'étude. Même si toutes les villes souhaitent améliorer leur compétitivité par le biais de tous les facteurs déterminés, le tableau attire l'attention sur le fait que chacune a des possibilités à mettre en valeur, pour peu que l'on sache penser le système urbain en réseau.

\section{Discussion des résultats}

La première hypothèse posée, selon laquelle les principales villes de la Beira Litoral assumeraient une prépondérance dans les hiérarchies établies se vérifie partiellement. En effet, parmi les dix premiers con celhos, plus de la moitié appartiennent à la sous-région de la Beira Litoral quant aux facteurs emploi, pouvoir d'achat, tourisme, qualification de la main-d'œuvre et services supérieurs, dynamisme des entreprises, effet d'échelle, internationalisation et urbanité. Pour ce qui est $\mathrm{du}$ facteur secteur tertiaire-consommation, les dix premières positions se répartissent également. C'est seulement en ce qui a trait à la sécurité urbaine et la sécurité routière qu'il y a un avantage pour la sous-région de la Beira Interior, surtout à cause de sa faible densité.

La seconde hypothèse, selon laquelle il existerait des spécialisations différentes par facteur de compétitivité dans les villes étudiées, est complètement vérifiée et permet aussi d'identifier les facteurs de compétitivité 


\begin{tabular}{|c|c|c|}
\hline 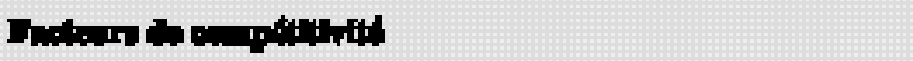 & 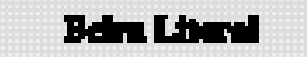 & 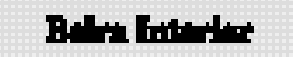 \\
\hline 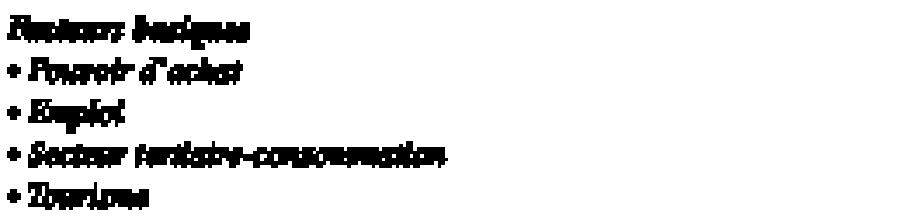 & 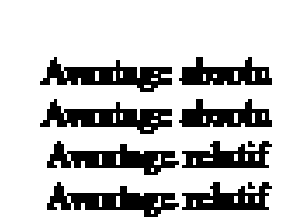 & مlif \\
\hline 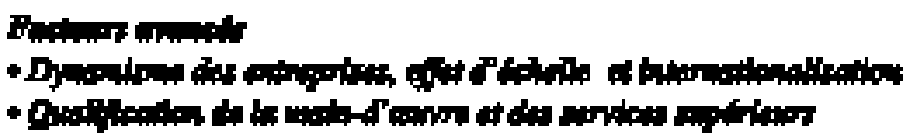 & 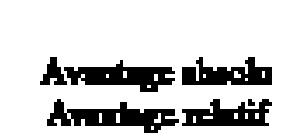 & 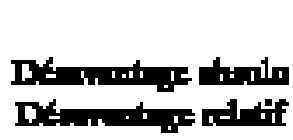 \\
\hline 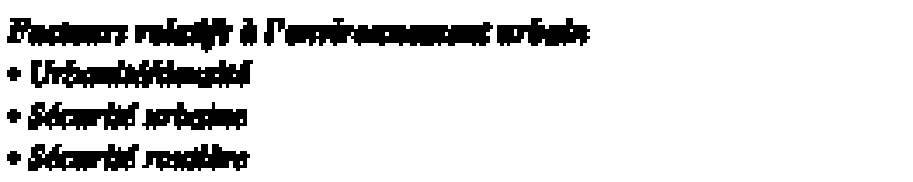 & 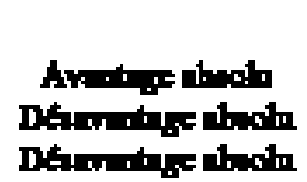 & 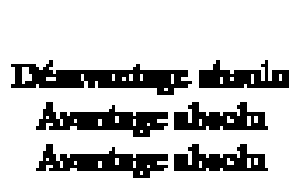 \\
\hline
\end{tabular}

sur lesquels les concelhos peuvent parier le plus efficacement.

La troisième hypothèse, selon laquelle il serait possible d'établir des avantages ou des désavantages absolus et comparatifs de l'ensemble des villes de Beira Litoral vis-à-vis l'ensemble des villes de Beira Interior et vice versa, est aussi confirmée et conduit à une identification des avantages et désa vantages absolus et relatifs de chaque ville-concelho.

Dans un scénario économique et social dominé par les nouvelles technologies de l'information, nous croyons que les territoires pourront être de plus en plus attractifs s'ils disposent d'une main-d'oeuvre qualifiée et de services supérieurs. Cette constatation pousse les concelhos de l'intérieur du Portugal à concentrer leurs investissements dans le développement des services supérieurs, à encourager la recherche appliquée pour produire de nouveaux contenus adaptés au paradigme des régions périphériques, à privilégier la santé et le loisir et à considérer comme moins prioritaire l'obtention d'économies d'échelle, en dépit du fait que ces économies demeurent importantes.

La viabilité des territoires de l'intérieur doit s'ancrer dans la combinaison des avantages actuels de régions de faible densité avec les avantages relatifs de connaissance (information moderne et mémoire collective riche).

Les villes-concelhos du littoral devront poursuivre leur parcours de développement sans oublier que la compétitivité territoriale doit être coopérative et complémentaire et non prédatrice. De cette façon, la rivalité entre le littoral et l'intérieur pourra se transformer en une coopération-compétition se déployant dans l'espace national, mais rejoignant aussi les espaces ibérique et européen.

\section{Notes et références}

1 Je tiens à remercier le Docteur Guedes de Carvalho pour m'avoir orienté et pour son amitié, sans quoi ce travail n'aurait pas été possible. Je veux également remercier le professeur Serge Côté, qui a revu le texte.

2 CAMAGNI, Roberto (1993). «Principi di Economia Urbana e Territoriale», La nuova Italia Scientifica, Roma.

3 LOPES, Simões Lopes (1971). As Funções Económicas dos Pequenos Centros, Fundação Caloust Gulbenkian, Centro de Economia e Finanças, Lisboa ; AYDALOT, Philippe (1986). Économie régionale et urbaine, Éditions Economica, Paris ; SALGUEIRO, Teresa Barata (1992). A cidade em Portugal : Uma geografia urbana, $2^{\mathrm{e}}$ Édition, Edições Afrontamento.

4 DOMINGUES, Álvaro (1996). «Política Urbana e Competitividade», Sociedade e território, Revista de Estudos Urbanos e Regionais, $\mathrm{n}^{\circ} 23$, octubro.

5 CAPELLO, Roberta (1998). «Économies d'échelle et taille urbaine : théorie et études empiriques revisitées», Revue d'Économie régionale et urbaine, $\mathrm{n}^{\mathrm{O}} 1$.

6 OLHIN, Bertil (1933). Interregional and International Trade, Havard University Press, Édition revue de 1967, Cambridge, Massachusetts.

7 PORTER, Michael (1993). A Vantagem Competitiva das Nações, Editora Campus, Rio de Janeiro. 